\title{
Performances of Clustered Ad Hoc Networks on 3D Terrains
}

\author{
Sonja Filiposka \\ Faculty of Electrical Engineering and \\ Information Technology \\ Karpos 2 bb \\ P.O. Box 574 \\ +38923099153 \\ filipos@feit.ukim.edu.mk
}

\author{
Dimitar Trajanov \\ Faculty of Electrical Engineering and \\ Information Technology \\ Karpos 2 bb \\ P.O. Box 574 \\ +38923099153 \\ mite@feit.ukim.edu.mk
}

\author{
Marija Vuckovik \\ Faculty of Electrical Engineering and \\ Information Technology \\ Karpos 2 bb \\ P.O. Box 574 \\ +38923099153 \\ marija_vuckovik@yahoo.com
}

\begin{abstract}
Propagation prediction algorithms which use terrain information are typically used for the design of modern wireless systems. Therefore, it is essential that when analyzing the performances of ad hoc networks we observe the network in a natural 3D terrain and we use an appropriate propagation model. In this paper we explain our extension of the NS-2 simulator using Digital Elevation Model data and terrain aware propagation model. Because of the natural emergence of clustering in the network of users, we also study the relationship between the clustering in the ad hoc networks and different terrain profiles. The simulations show that, when the network clusters are accompanied with related terrain contours, the observed network performances demonstrate measurable enhancement.
\end{abstract}

\section{Categories and Subject Descriptors}

C.2.1 [Network Architecture and Design]: Wireless communication

\section{General Terms}

Algorithms, Performance, Design, Experimentation, Theory, Verification.

\section{Keywords}

3D terrain data, Durkin's propagation model, ad hoc networks, clustering, performances

\section{INTRODUCTION}

The ability to communicate with people on the move has evolved remarkably during the last decade. The mobile radio communications industry has grown by orders of magnitude and made portable radio equipment smaller, cheaper and more reliable [1]. The large scale deployment of affordable, easy-to-use radio communication networks has created a trend of a demand for even

Permission to make digital or hard copies of all or part of this work for personal or classroom use is granted without fee provided that copies are not made or distributed for profit or commercial advantage and that copies bear this notice and the full citation on the first page. To copy otherwise, or republish, to post on servers or to redistribute to lists, requires prior specific permission and/or a fee.

SIMUTools 2009, March 2-6, Rome, Italy.

Copyright 2009 ICST, ISBN 978-963-9799-45-5. greater freedom in the way people establish and use the wireless communication networks [23].

One of the consequences to this ever present demand is the rising popularity of the ad hoc networks. A mobile wireless ad hoc network (MANET) is an infrastructure-less network that can be established anywhere on the fly [2]. It consists of wireless mobile nodes that communicate directly without the use of any access point or base station. Thus, the nodes are supposed to establish a network environment by the means of self organization in a highly decentralized manner. In order to achieve this goal every node has to support the so-called multihop paths. The multihop path concept is introduced to allow two distant nodes to communicate by the means of the intermediate nodes to graciously forward the packets to the next node that is closer to the destination. This is controlled by a special ad hoc routing protocol [3] that is concerned with discovery, maintenance and proper use of the multihop paths.

The independence of existing infrastructure, as well as the ability to be created instantly, that is, on demand, has made the ad hoc networks a very convenient and irreplaceable tool for many onthe-go situations like: rescue teams on crash sites, vehicle to vehicle networks, lumber activities, portable headquarters, late notice business meetings, military missions, and so on. Of course, every one of these applications demands a certain quality of service from the ad hoc network and usually the most relevant issue are the network performances in terms of end-to-end throughput. However, the tradeoff of having no infrastructure and no centralized manner of functioning has influenced the ad hoc networks performances greatly on many aspects.

As for all wireless mobile communications, maybe here more that for others, the mobile radio channel places fundamental limitations on the performances of the ad hoc network. Radio transmission in a mobile communications system often takes place over irregular terrain. Therefore, the terrain profile of a particular area needs to be taken into account for estimating the path loss. The transmission path between the transmitter and the receiver can vary from simple line-of-sight to one that is severely obstructed by buildings, hillsides or foliage.

Modeling the radio channel has historically been one of the most difficult parts of mobile radio system design. Propagation models have traditionally focused on predicting the average received signal strength at a given distance from the transmitter, as well as the variability of the signal strength in close spatial proximity to a particular location. 
Typically when estimating the ad hoc network performances very little attention is given to the terrain, and thus, to the propagation model used for the estimation [2][4][5]. The nodes are assumed to be scattered on a flat, regular terrain and a simple ground reflection propagation model is used. However, since terrain irregularities can greatly affect and distort the expected network performances, great care must be taken when trying to picture the real-life use of the modeled network.

In order to bring our observations a large step closer to the reallife ad hoc network deployment, we decided to use a propagation model that incorporates the nature of propagation over irregular terrain and losses caused by obstacles in the radio path. We created an implementation of the Durkin's model [6] as an extension for the NS-2 simulator [7], thus allowing us to conduct more realistic simulation scenarios and analyze the way the terrain profile affects the ad hoc network performances.

Since the model is based on terrain information extracted from a geographic information system file format, we also created a tool that can be used to create terrains in the given format by simple description of the terrain characteristics using a given number of triangular planes. In this way we can model fairly simple, but also very rich detailed terrains and avoid the necessity of using realworld data, since the appearance of the real-world data can not be controlled in a satisfactory manner.

Another natural phenomenon that we wanted to incorporate in our ad hoc networks studies is the clustering on both, logical and physical, layer. In order to do so we first investigated the properties of the networks that describe the relationships between the network users. It has been shown that social networks exhibit several interesting characteristics that have been incorporated in the theory of small world networks [19], [20]. One of the special features of small world networks is the high clustering of the nodes which is naturally occurring because of the peoples tendency to form groups of friends that share strong ties and keep weak and seldom connections to people that do not belong to their group [21]. This social behavior directly affects the ad hoc network because we can easily empathy the wireless mobile nodes with the social actors. The user of the network and his needs and affinities are driving the main objectives of the ad hoc network node including the physical positioning and communication (logical) pattern [22]. Hence, when studying ad hoc networks we incorporate the social clustering in the way we model the physical positioning of the nodes (friends tend to stay close to friends) and the model of the communication pattern on application layer (friends tend to share information with friends) [24].

In this paper the first order analysis of clustered ad hoc network performances when deployed on 3D terrains using an appropriate propagation model are given. The rest of this paper is organized as follows. In Section 2 an introduction to the digital elevation model file format is given as well as the description of the tool we created for construction of artificial terrains that are described in this file format. Section 3 describes the Durkin's irregular terrain propagation model and the way we implemented it in the NS-2 simulator. In Section 3 the simulation scenarios are specified with an accent to the way we modeled the clustering of the network and in Section 4 the analysis of the clustered ad hoc network performances in 3D terrains are presented. Finally, Section 5 concludes this paper.

\section{TERRAIN MODEL}

The Geographic Information System (GIS) [8] integrates hardware, software and data for storing, management, analysis and representation of all forms of geographically referenced data. GIS technology [9] can be used for science purposes, cartography, urban planning, rescue missions and everywhere where need for geospatial parameters of the earths surface arises. GIS files are defined according to very strict standards for keeping record of digitalized data. GIS files contain elevation data, terrain shape, coordinate systems and projections and one or more data that describe the precise shape of the earth's surface according to the given coordinates.

The geographic systems are differentiated according to the way they store the elevation data. There are two formats for data storing: raster and vector. The raster type of data consists of rows and columns of cells wherein a unique value is stored. Each cell gets a numeric value that can be represented by a unique identifier. The resolution of the raster data is the cell width and length in earth units. Usually the cells are square terrain areas, but other shapes can also be used.

The USGS DEM standard [10] is a geospatial file format developed by United States Geological Survey (USGS) for storing raster based digital elevation models (DEM). The USGS DEM file [11] is a set of ASCII coded blocks with size of $1024 \mathrm{~B}$. The file is organized in three categories of records labeled with A, B and C.

Record A contains information for the origin and type of the terrain, as well as summary characteristics and measurement systems, which are used by the profiles. The profiles (records B) are columns with arbitrary length that contain raster elevations beginning at a certain location. Record $\mathrm{C}$ contains the standard deviation as a quality control data, as well as other statistics about the data precision.

\subsection{Terrain Generator}

There are several software packets that allow the user to create an artificial terrain in the DEM file format like Terraform [12] and Terragen [13]. However, when using these applications, the user has no direct impact on the terrain shape, but as input parameters he can only give the statistical characteristics of the desired terrain like the average height and the deviation from the average height in the sense of a global terrain contour (i.e. hill type or plain type). Our idea was to be able to give the precise shape of the desired terrain so as to completely control the outcome.

For these purposes we developed a special tool for terrain generation. The tool provides terrain generation based on the input given by the user. The user needs to define the desired terrain by the means of a number of triangular planes. The input data for the terrain generator are the three coordinates of each triangular plane. The input is defined using an external XML file with the following specification:

- $\quad<$ PLANE $>$

$\begin{array}{ll}\circ & <\text { No. }> \\ \circ & <\text { NAME }> \\ \circ & <\text { ENDPOINTS }>\end{array}$




$$
\begin{aligned}
& \text { - } \quad<\text { No. }> \\
& \text { - } \quad<\mathrm{X}>,<\mathrm{Y}>\text {, and }<\mathrm{Z}>\text {. }
\end{aligned}
$$

The terrain is generated into a 7.5-minute DEM file, see Fig. 1. The maximum and minimum heights of the terrain are calculated from the given input. The terrain data is stored relatively to the minimum height. The resolution of the terrain is 10:10:0.1 (in meters) as best given by the 7.5-minute standard.

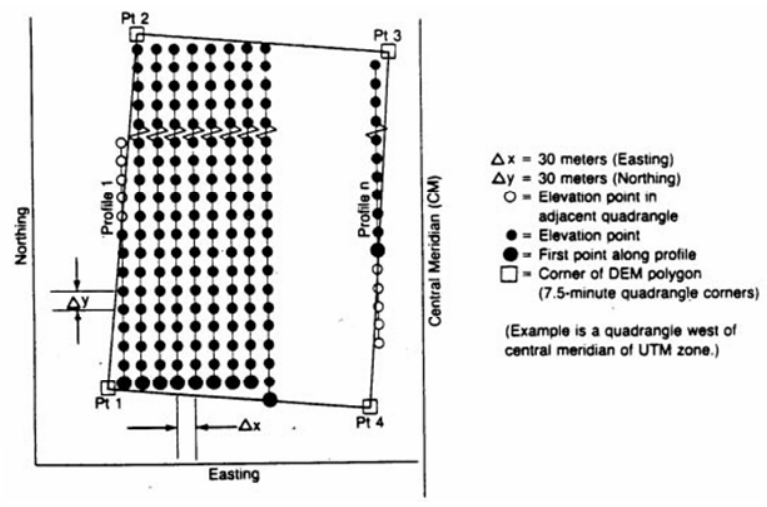

Figure 1. Extracting values for a 7.5 minute DEM file

Our artificial terrain generator allows us to create various types of terrains, from simple designed terrains consisting of several flat surfaces, up to a rich detailed terrain that can closely resemble a real world terrain data. This possibility allows us to fine tune the simulation environment, that is, to create a terrain that perfectly fits to the needs of the simulation. Thus, we gain more flexibility compared to real terrains for which you first need to find a terrain that has the wanted features and then cut it from the DEM file that contains it (the freely available DEM files are usually for very large areas) and create a new DEM file with the wanted piece which is a time consuming task. The terrain generator also creates the possibility to investigate how much exactly the terrain data influences on the network performances when thinking in terms like: "Are the rough terrain features the ones responsible for the observed network behavior or the small terrain discrepancies also play a big role in the shaping of the communication paths?". This can be done by using a different level of details when generating the terrains for the simulations and is one of our planned future steps in our study.

\subsection{NS-2 nodes in 3D Space}

Using our terrain generator we are able to create various artificial terrains that can afterwards be read in the NS-2 simulator using the specialized DEMfile class which is already part of the NS-2 simulator. This class is one of the starting points for transition to 3D simulations. However, in the several last versions of this simulator there is still no progress regarding the transition from 2D flat space to 3D space defined by the means of a DEM file. Using the existing class, that needed a few adjustments to work properly, we were able to read in a given DEM file and fill out the topographical database that will keep the terrain profiles needed for simulation.

The topographical database can be thought of a two-dimensional array. Each array element corresponds to a point in the simulated area map, while the actual contents of each array element is the elevation above sea level data.

The next step was making adjustments in the way NS-2 treats the node coordinates, since although when the classes were built it was thought to eventually transfer to $3 \mathrm{D}$ space, the functions always ignore the third coordinate for elevation. We redid the appropriate functions that were supposed to return the actual $\mathrm{Z}$ coordinate of the node in the $3 \mathrm{D}$ space by the means of calculating the height of the ground level for the given $\mathrm{X}$ and $\mathrm{Y}$ coordinates of the node using the topographical database. With these changes, the NS-2 simulator was translated into the $3 \mathrm{D}$ space.

Another fine adjustment was adding the possibility to regulate the scale of the terrain given in the DEM file. This is due to the fact that if we decide to use real terrains, the ones that are publicly available using the Internet are usually very large and thus not applicable for small and medium size wireless network simulations. Our solution offers the user to read in a DEM file that describes a large terrain and then scale it to a desired size inputted by the user in the tcl script that describes the simulation scenario. In the input tcl script that describes the simulation scenario the desired width and length of the terrain is given. Using these values, after reading in the given DEM file, we scale the data using the new values for terrain dimensions. This feature also allows us to make the resolution of our terrain much higher than the 10:10:1 as given by the standard. Thus, the accuracy can be set to $1 \mathrm{~m}$ or even less.

Our idea was to insert 3D data into the simulator and change the way the simulator checks for line-of-sight events or for existence of some obstacles between the transmitter and receiver. Thus, on this level where we have the terrain data we also created a number of functions that can be used to check the terrain characteristics in a given area. For an example, we have function that returns an interpolated height of the terrain for a given point and range for interpolation. We also have functions that check the existence of obstacles for a given ellipse which are later used for Fresnel zone clearance determination. Of course, there is a function that determines whether there is line of sight between two points on the terrain. The existence of these functions that are based on geometrical projections and transformation makes it very easy to add on another layer that can use them in order to decide, for example, the level of the received signal for a given node. We used the functions in our implementation of the Durkin's propagation model in NS-2, but they are also made reusable for an implementation of a different propagation model or some other part of the simulator that might need some geometry data about the terrain.

\section{DURKIN'S PROPAGATION MODEL}

When simulating wireless mobile networks habitually we come to use one of the large-scale propagation models that estimate the radio coverage area of a transmitter for an arbitrary transmitterreceiver separation distance [12]. These practical and fast, yet terrain unaware, frequently used propagation models are the free space propagation model or the ground reflection (two-ray) propagation model.

The free space propagation model is used to predict received signal strength when the transmitter and receiver (T-R) have a 
clear, unobstructed line-of-sight path between them. The free space power received by a receiver antenna which is separated from a radiating transmitter antenna by a distance $d$, is given by the Friis free space equation,

$$
P_{r}(d)=\frac{P_{t} G_{t} G_{r} \lambda^{2}}{(4 \pi)^{2} d^{2} L}
$$

where $P_{t}$ is the transmitted power, $P_{r}(d)$ is the received power which is a function of the T-R separation, $G_{t}$ is the transmitter antenna gain, $G_{r}$ is the receiver antenna gain, $d$ is the T-R separation distance in meters, $L$ is the system loss factor not related to propagation $(L \geq 1)$, and $\lambda$ is the wavelength in meters.

In a mobile radio channel, a single direct path between T-R is seldom the only physical means for propagation, and hence the free space propagation model of (1) is in most cases inaccurate when used alone. The two-ray ground reflection model shown in Fig. 2 is a useful propagation model that is based on geometric optics, and considers both the direct path and a ground reflected propagation path between T-R. This model has been found to be reasonably accurate for line-of-sight microcell channels.

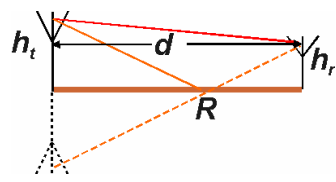

Figure 2. Two-ray ground reflection model

The received power at a distance $d$ from the transmitter for the two-ray ground bounce model can be expressed as

$$
P_{r}=P_{t} G_{t} G_{r} \frac{h_{t}^{2} h_{r}^{2}}{d^{4}}
$$

where $h_{t}$ is the height of the transmitter and $h_{r}$ is the height of the receiver. As seen from (2) at large distances $\left(d \gg \sqrt{h_{t} h_{r}}\right)$, the received power falls off with distance raised to the fourth power, or a rate of $40 \mathrm{~dB} / \mathrm{decade}$. This is much more rapid path loss than is experienced in free space.

One of the basic mechanisms of radio propagation is diffraction that causes radio signal distortions. Diffraction allows radio signals to propagate around the curved surface of the earth and to propagate behind obstructions. Although the received field strength decreases rapidly as a receiver moves deeper into the obstructed (shadowed) region, the diffraction field still exists and often has sufficient strength to produce a useful signal.

The concept of diffraction loss as a function of the path difference around an obstruction is explained by Fresnel zones. Fresnel zones represent successive regions that have the effect of alternately providing constructive and destructive interference to the total received signal. The radius of the nth Fresnel zone circle is denoted by $r_{n}$ and can be expressed in terms of $n, \lambda, d_{1}$ and $d_{2}$ by

$$
r_{n}=\sqrt{\frac{n \lambda d_{1} d_{2}}{d_{1}+d_{2}}}
$$

where $d_{1}$ and $d_{2}$ are distanced from the obstacle to the transmitter and the receiver, respectively.
In mobile communication systems, diffraction loss occurs from the blockage of secondary waves such that only a portion of the energy is diffracted around an obstacle. That is, an obstruction causes a blockage of energy from some of the Fresnel zones, thus allowing only some of the transmitted energy to reach the receiver. Depending on the geometry of the obstruction, the received energy will be a vector sum of the energy contribution from all unobstructed Fresnel zones.

In general, as shown on Fig. 3, if an obstruction does not block the volume contained within the first Fresnel zone, then the diffraction loss will be minimal, and diffraction effects may be neglected. In fact, as long as $60 \%$ of the first Fresnel zone is kept clear, the further Fresnel zone clearance does not significantly alter the diffraction loss.

When shadowing is caused by a single object such as a hill or mountain, the attenuation caused by diffraction can be estimated by treating the obstruction as a diffracting knife edge. This is the simplest of diffraction models, and the diffraction loss in this case can be readily estimated using the classical Fresnel solution for the field behind a knife edge.

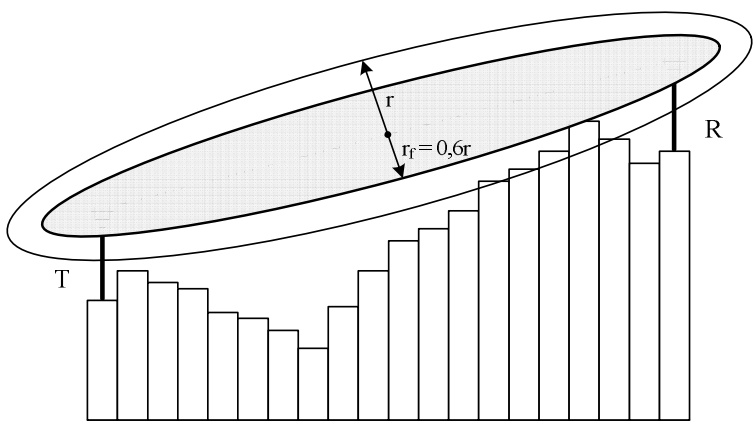

Figure 3. Fresnel zones and diffraction loss due to zone blockage

The diffraction gain due to the presence of a knife edge, as compared to the free space, is given by

$$
G_{d}(\mathrm{~dB})=20 \log |F(v)|
$$

where $F(v)$ is the Fresnel integral, a function of the FresnelKirchoff diffraction parameter $v$, defined as

$$
v=h \sqrt{\frac{2\left(d_{1}+d_{2}\right)}{\lambda d_{1} d_{2}}}
$$

where $h$ is the relative height of the obstruction. In practice, graphical or numerical solutions are relied upon to compute the diffraction gain. An approximate solution for (4) provided by [11] is

$$
\begin{array}{lrl}
G_{d}(\mathrm{~dB})=0 & v \leq-1 \\
G_{d}(\mathrm{~dB})=20 \log (0.5-0.62 v) & -1 \leq v \leq 0 \\
G_{d}(\mathrm{~dB})=20 \log \left(0.5 e^{-0.95 v}\right) & 0 \leq v \leq 1 \\
G_{d}(\mathrm{~dB})=20 \log \left(0.4-\sqrt{0.1184-(0.38-0.1 v)^{2}}\right) & 1 \leq v \leq 2.4 \\
G_{d}(\mathrm{~dB})=20 \log \left(\frac{0225}{v}\right) & v>2.4
\end{array}
$$




\subsection{Durkins's Model}

The median transmission loss predicted using the path geometry of the terrain profile is the goal of the Edwards and Durkin [5], as well as Dadson [12]. Our implementation of this propagation model in the NS-2 working environment closely follows their concepts.

The execution of the path loss estimation consists of two parts. The first part addresses a topographic DEM file turned into a topographical database (see Section 2) and reconstructs the ground profile information along the path between T-R. The second part of the algorithm calculates the expected path loss along that path.

Since the T-R path may not always pass through discrete data points, interpolation methods are used to determine the approximate heights that are observed when looking along the path. Fig. 4 shows the T-R path and the points with which to use diagonal linear interpolation. Fig. 3 shows what a typical reconstructed terrain profile might look like. Each point of the reconstructed profile consists of an average of the heights obtained by diagonal, vertical and horizontal interpolation methods. This means that we move along the line that connects T$\mathrm{R}$ in discrete steps. The width of the step can be adjusted by the user, by default is $1 \mathrm{~m}$. In each step, for interpolation we consider the horizontal, vertical and diagonal cells that are in a given range (also set by the user) around the point on the T-R line.

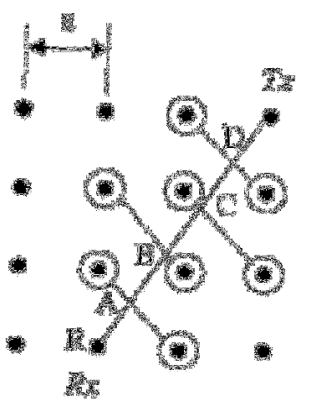

Figure 4. Horizontal, vertical and diagonal interpolation

At this point the algorithm must make decisions as to what the expected transmission loss should be. The first step is to decide whether a line-of-sight (LOS) path exists between T-R. To do this, we compute the difference $\delta$ that is the height of the line joining $\mathrm{T}-\mathrm{R}$ antennas from the height of the ground profile in $3 \mathrm{D}$ space.

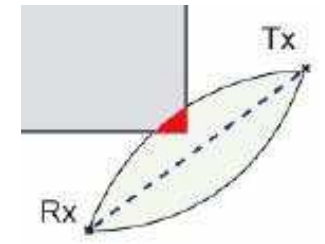

Figure 5. Considering the Fresnel zone in 3D space with the "sides"

Since we are using the propagation model for modeling wireless mobile networks it is essential that we consider the Fresnel zone in 3D, see Fig. 5. This means that we consider potential penetration of the Fresnel zone not only in the $\mathrm{Z}$ plane, but also in its "sides". This additional 3D ellipse observation is not done in the original Durkin's model.
If any $\delta$ is found to be positive, it is concluded that a LOS path does not exists, otherwise it can be concluded that a LOS path exists. Assuming the path has a clear LOS, we check to see whether first Fresnel zone clearance is achieved. If the Fresnel zone of the radio path is unobstructed, then the resulting loss mechanism is approximately that of free space. If there is an obstruction that just barely touches the line joining the transmitter and the receiver then the signal strength at the receiver is $6 \mathrm{~dB}$ less than the free space value due to energy diffracting off the obstruction and away from the receiver. The method for determining first Fresnel zone clearance is done by first calculating the Fresnel diffraction parameter $v$, defined in (5), for each of the $j$ ground elements.

If $v_{j} \leq-0.8$ for all $j=1, \ldots, n$, then free space propagation conditions are dominant. For this case, the received power is calculated using the free space transmission formula given in (1). If the terrain profile failed the first Fresnel zone test (i.e. any $\left.v_{j}>-0.8\right)$, then there are two possibilities:

\section{- $\quad$ Non-LOS}

- LOS, but with inadequate first Fresnel zone clearance

For both of these cases, the program calculates the free space power using (1) and the received power using the plane earth propagation equation given by (2). The algorithm then selects the smaller of the powers calculated with (1) and (2) as the appropriate received power for the terrain profile. If the profile is LOS with inadequate first Fresnel zone clearance there is loss that is added (in $\mathrm{dB}$ ) to the appropriate received power. This additional diffraction loss is calculated by (6).

The loss is evaluated using the highest value of the diffraction parameter $v$, which means that we settle for the worst case of all existing diffraction knife edges. For the case of non-LOS, the system grades the problem into the category of single diffraction edge. This is a first step simplification of the procedure, that in the future version of the software we intend to expand and correct so that we check for two, three and more diffraction edges using the Epstein and Peterson method [13].

\subsection{Durkin's Model Implementation in NS-2}

In order to apply the Durkin's model in the NS-2 simulation environment, we created a new type of propagation model Durkin, which is based on the previously described algorithm.

The antenna height (in 3D space), the carrier frequency of the radio signals and node position in $3 \mathrm{D}$ space are input parameters to the propagation model. The overall node elevation is sum of terrain elevation and antenna height.

The check for first Fresnel zone clearance is made in a parameterized manner so that the user can control the necessary percent of clearance of the first Fresnel zone.

As explained earlier, if the first Fresnel zone of the radio path is unobstructed the expected signal loss calculation is according to the free space propagation model. If the difference is smaller than the given percent of the radius of the first Fresnel zone we determine that there is a breach in the zone. At the moment of loss of line of sight the procedure calculates the loss due to the diffraction losses. 
We also had to modify the CSMA/CA channel in NS-2 so it can determine the presence of the terrain, i.e. line of sight. The current design of the NS-2 wireless channel considers square shape of the physical carrier sense range of the transmitting node. Hence, the nodes which are in the range of that square are not able to access the channel. Using our propagation model results in a physical carrier sensing ranges of the transmitting node, similar to its real world omni-directional spherical shape when there are no obstructions near the node.

Nevertheless, the transmission and physical carrier sensing ranges of the signal propagation of the nodes are not ideal circles, but they depend on the terrain configuration. The situation without terrain assumes that some nodes might be blocked by other sending nodes for example the hidden and exposed terminal problems. Considering the terrain as another parameter implies that some obstacles might limit the interferences between the nodes. In this way, the resources of the channel are more utilized and the hidden and exposed terminal problems are reduced.

For our first set of investigation we settled for a single knife edge diffraction in order to lessen the computational burden that was introduced to the simulator. By the means of a software profiling tool we analyzed the performances of our new class for the Durkin's propagation model and found that the repeated accessing to the topographic database which is held in memory as a dynamic one-dimensional array is the most time consuming part because it is a function call that is used around 10 times more than any other call in the algorithm. Also, the other calculations mainly consist of time consuming functions like square roots and logarithms that also add to the time needed for processing one pair of transmitting receiving nodes. When taking into consideration that in NS-2 this is done in a way that for each transmitting node the receiving power level at each other node that is part of the network has to be calculated in order to decide whether there is interference or no, we came across a performance problem since the simulations were taking a tremendous amount of time.

In order to lessen the computing time, at the beginning we discard the 'impossible' situations, that is we immediately calculate the receiving power according to the free space propagation model and if it is below the threshold we have no need to check whether the signal is even less stronger than our first order optimistic approximation.

Another performance enhancement is introducing a cache in the case of simulation of static nodes. Since for static simulations the once already calculated received powers for a given transmitter can not change, we decided to keep these values in a cache in a form of a $N$ x $N$ matrix where $N$ is the number of nodes in the simulation. At the beginning of the algorithm we first check to see whether we already have the necessary values in the cache. If they are available we simply reuse them, otherwise we calculate and add the new values in the cache. In this way, the speed of completing our simulation sets was brought back to the original when simulating in the usual $2 \mathrm{D}$ environment.

As for the cases of mobile nodes, we are not able to use cache because of the enormous number of possibilities. (If we use a
$500 \mathrm{~m} \times 500 \mathrm{~m}$ terrain with a resolution of $1 \mathrm{~m}$, we have $25 \times 10^{4}$ combinations for only one position of the transmitter and totally $\sim 10^{9}$ combinations for every position of the transmitter. Using 32 bits for storing the resulting received power we need $32 \mathrm{~GB}$ for all of the possibilities. This number can be reduced several times, but it will still be too large for any practical use.) Thus, for this types of simulations the time for execution of the $3 \mathrm{D}$ simulation is several times greater than the $2 \mathrm{D}$ type.

\section{SIMULATION SCENARIOS}

The primary objective of our simulations is to understand the impact of the terrain presence in a simulation environment for clustered ad hoc networks. To this end, we evaluate the aspects of traffic throughput of the Durkin propagation model compared with the traditional 2D flat terrain approach using the two-ray ground propagation model. In the simulation we are utilizing the AODV protocol [14] for route discovery and path set up as well as the UDP protocol for data packets sending so that we do not have to deal with the TCP overhead.

For our first series of evaluations made in this paper, we decided to work with fairly simple artificial terrains in the shape of a hillside, hill, ravine and pyramid. On Fig. 6 (a, b, c and d) the terrain shapes are shown using the DEM file visualizing software 3 Dem. For comparison and verification purposes we use the results obtained for a perfectly flat terrain, for which the Durkin model is broken down to the usual two-ray ground propagation model. In this way we can determine the terrain features impact on the network performances.

\subsection{Simulation Environment}

The created DEM files we used for the simulations were made with dimensions of $10.000 \mathrm{~m} \times 10.000 \mathrm{~m}$ with a $10: 10: 1$ resolution. We used the scaling feature we implemented in the NS2 simulator in order to obtain precision of $1 \mathrm{~m}$. Thus, the simulation area for all terrains is to $1000 \mathrm{~m} \times 1000 \mathrm{~m}$ with highest point of $200 \mathrm{~m}$. We have 100 static nodes that are uniformly dispersed in the simulation area. The node transmission range is the standard $250 \mathrm{~m}$ (for two ray ground propagation model) given by the use of the IEEE $802.11 \mathrm{~b}$ standard wireless equipment. The antenna height is set to $1.5 \mathrm{~m}$ and it has no relative offset against the wireless node. The offered network load is varied from 0.1 to 7 Mbps using UDP data packets with $1 \mathrm{~KB}$ size.

The small world clustering characteristics of the ad hoc network are implemented on two levels independently. The first type of clustering is introduced via the Small World application layer as described in [15]. The complete ad hoc network of 100 nodes is logically represented with a small world network consisting of four groups each containing 25 nodes. In order to obtain the desired clustering coefficient we set the degree of each node to 6 . The way these connections are distributed inside and outside the node's group is controlled via the percent of in-cluster communication which for our simulation sets is set to $72 \%$ as this value represents an expected value for real-life situations [16]. The clustering character of the physical level of the ad hoc network is represented with four clusters of 25 static nodes. 


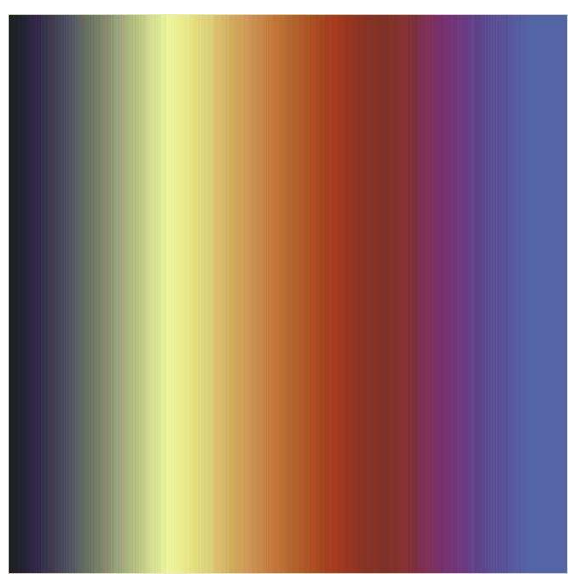

a) hillside terrain

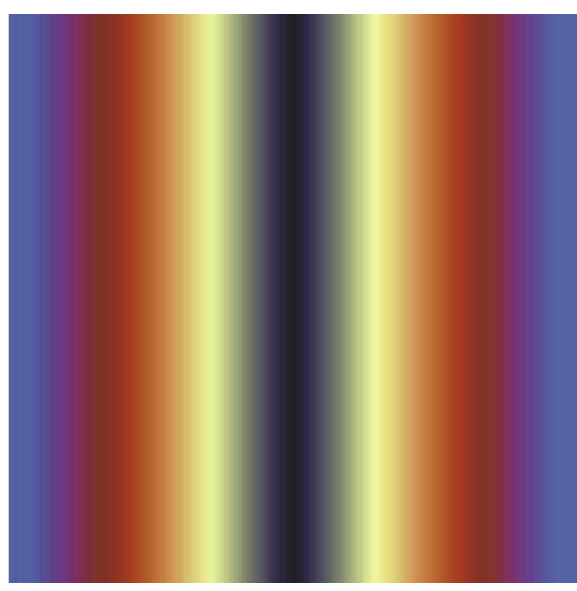

c) ravine terrain

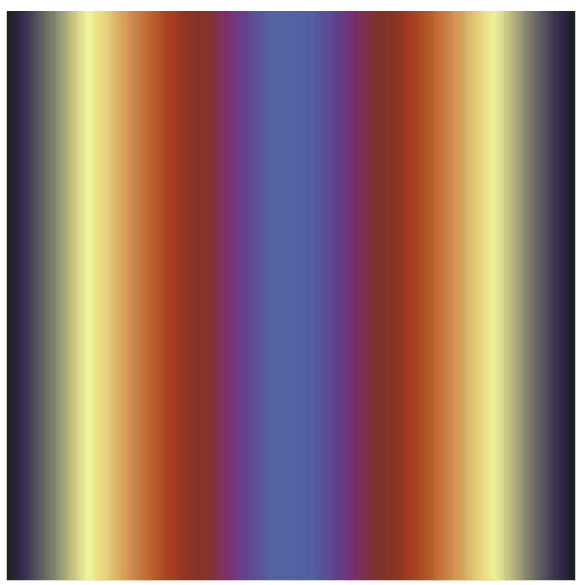

b) hill terrain

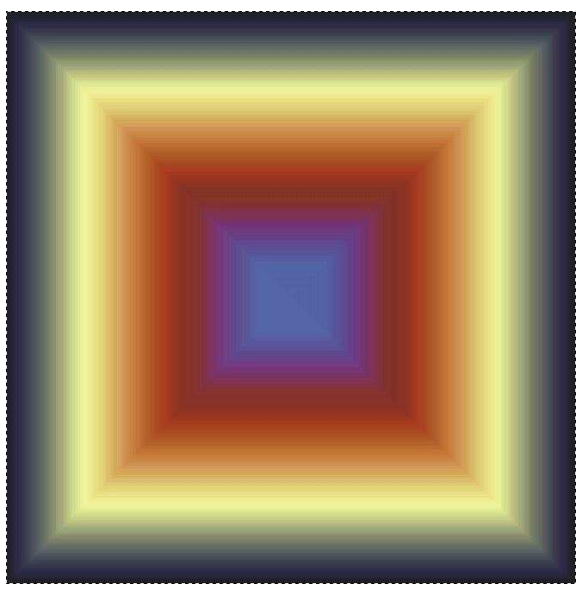

d) pyramid terrain

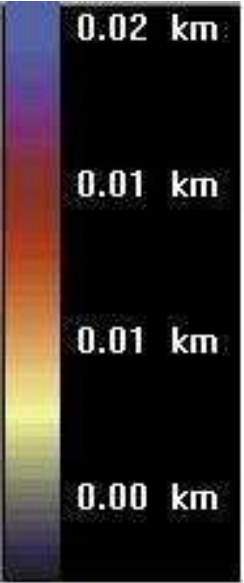

scale

Figure 6. Terrains generated with our terrain generator and used for the clustered ad hoc networks performance analysis

Please note that the grouping on the physical level corresponds to the grouping on logical level, namely, we have the same four groups of nodes. The square $\mathrm{XY}$ projection of the terrain is divided into 4 identical square parts with size 500m x 500m. Each ad hoc cluster has its own terrain part of the whole simulated area and the nodes that belong to the group are uniformly scattered in their belonging area part.

Consequently, from the clustering perspective, we have four different simulation settings, which are:

- $\mathrm{L}=0, \mathrm{P}=0$ - no clustering on application layer and no clustering on physical layer; the nodes are randomly scattered in the whole area and the communication is completely randomized

- $\mathrm{L}=1, \mathrm{P}=0$ - clustering on application layer only; the nodes are randomly scattered in the whole area, but the communication follows a small world pattern

- $\mathrm{L}=0, \mathrm{P}=1$ - clustering on physical layer only; the nodes are clustered into four groups, but the communication pattern is completely random
- $\quad \mathrm{L}=1, \mathrm{P}=1$ - clustering on both application and physical layer; the nodes are physically dived into four groups and the communication pattern is small world. Take into consideration that these two types of groups (application and physical) are overlapping as this is the most expected case.

\section{PERFORMANCE ANALYSIS}

One of the goals of this paper is to investigate the impact of the terrain features on the ad hoc network performances in the different cases of clustering.

As a comparison we use the results obtained when the ad hoc network is simulated over a flat terrain, see Fig. 7. The results clearly show that the clustered case, on logical and physical level, has outstanding performances over the other cases. It can also be concluded that the clustering on logical (application) layer has greater impact on the performance rise than that on physical layer.

The simulations that were done on a hillside terrain (Fig. 8.) show little performance enhancement (1.2 Mbps compared to $1 \mathrm{Mbps}$ for the flat terrain case) that can be more easily viewed for the 


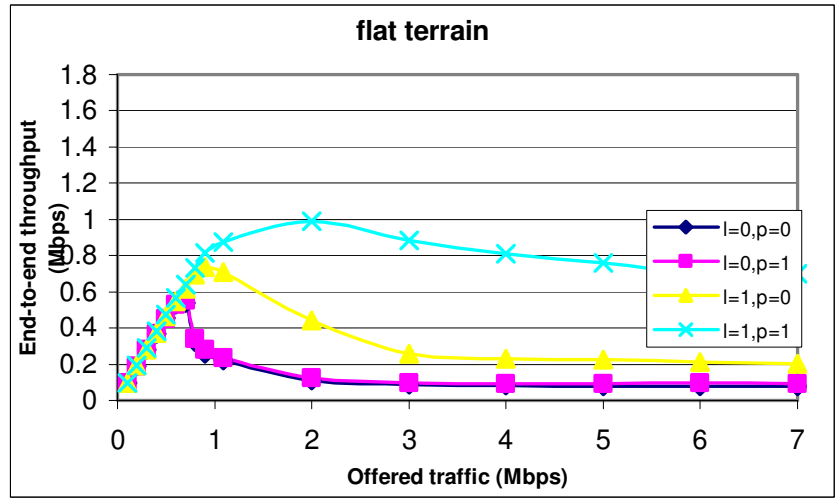

Figure 7. Performances of (non-)clustered ad hoc network on flat terrain area

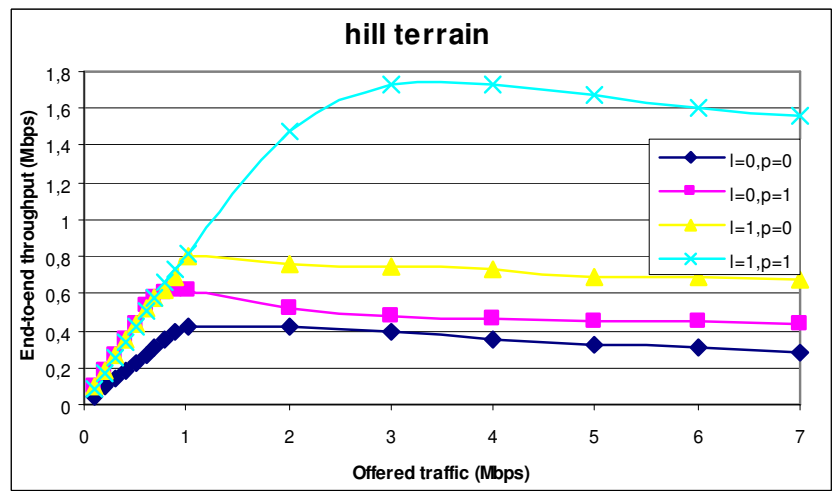

Figure 9. Performances of (non-)clustered ad hoc network on hill terrain area

logical and physical clustering case. This is due to the case when the groups on lower ground fail to cause interference to the groups on higher ground because the Fresnel zone is being blocked by the ground itself. However, for very high network loads the performances of the network are the same as for the flat terrain.

When viewing the obtained results for the hill terrain (see Fig. 9.), one can instantly observe change in the behavior of the network. Firstly, for all cases the network becomes more resilient to the increasing of network load when compared to the two previous cases. Also it is interesting to note that the maximum performance is reached for a higher load ( $3 \mathrm{Mbps}$, compared to $1 \mathrm{Mbps}$ ). For this case the physical clustering has a bit greater impact, which adds up to a max of $1.8 \mathrm{Mbps}$ end-to-end throughput for the $\mathrm{L}=1$ $\mathrm{P}=1$ case. The increasing performances for this terrain are a direct consequence of the terrain profile. The hill divides the complete area into two parts (two separate hillsides). In this way, the interference between the two parts of the hillsides is greatly lessened. The small world effect of the communications means rare communication between the two hillsides which are the hardest for establishing.

The ravine terrain (Fig. 10.) shows somewhat the reverse situation of the hill. The nodes are placed in a "hole" (see Fig. $6 \mathrm{c}$ ) in which the interference is even higher than usual (flat terrain)

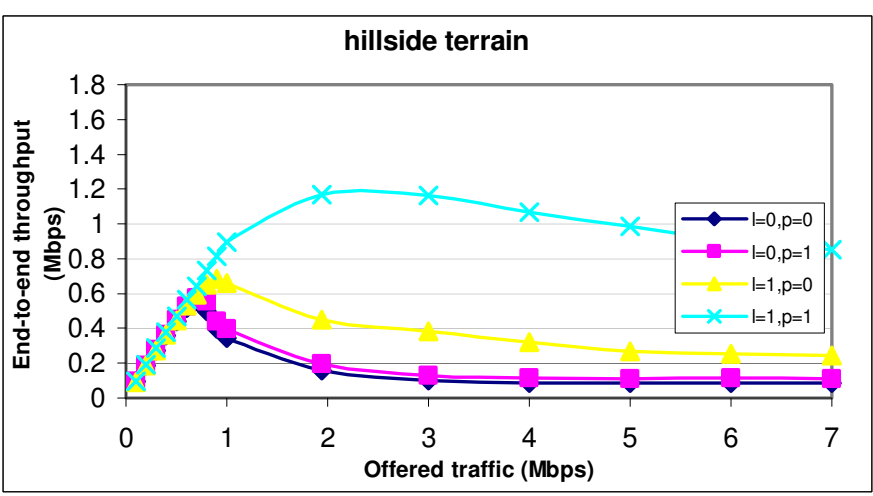

Figure 8. Performances of (non-)clustered ad hoc network on hillside terrain area

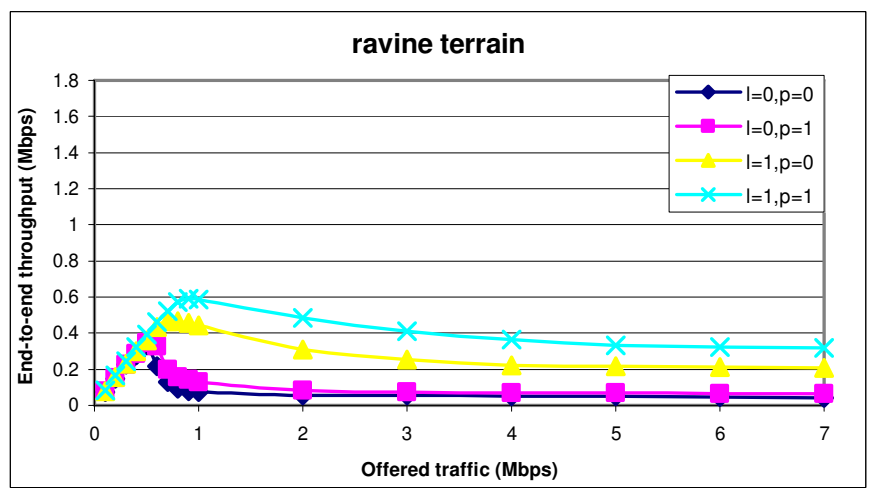

Figure 10. Performances of (non-)clustered ad hoc network on ravine terrain area

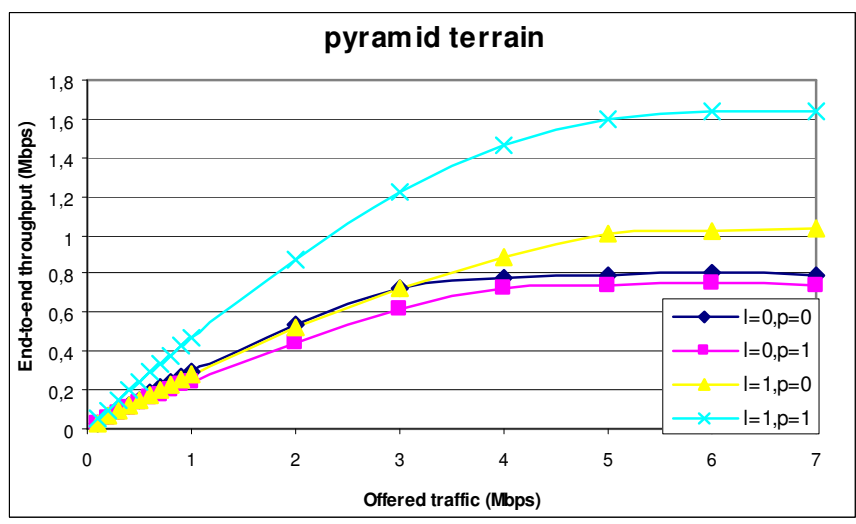

Figure 11. Performances of (non-)clustered ad hoc network on pyramid terrain area

because of the lack of terrain to block and reflect. The performances are degraded for about $50 \%$ compared to the ones obtained for a flat terrain. Again the logical clustering has a much higher impact than the physical clustering since it lessens the great interference between the nodes. Please note, however, that the Durkin's propagation model is a little bit pessimistic for this type of terrain configuration since it does not take into account any other reflection than the one from the ground. 


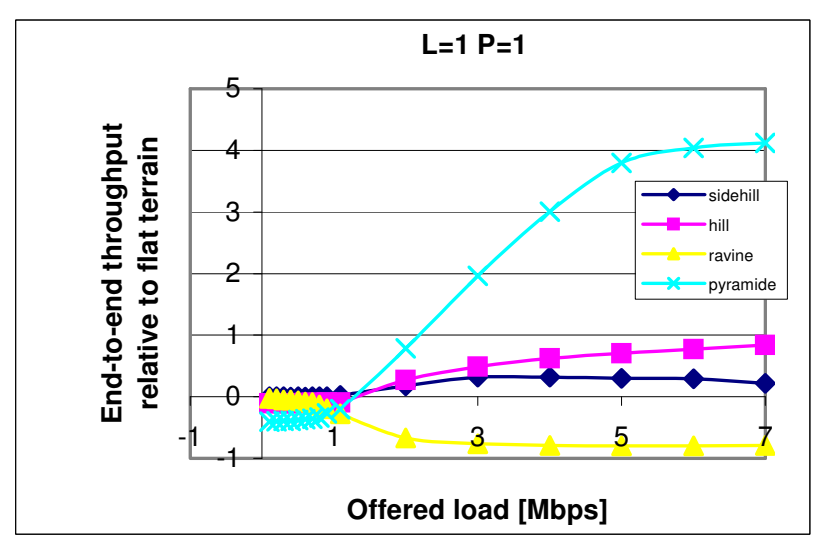

Figure 12. Relative performances of clustered ad hoc networks when compared to the performances for flat terrain

On Fig. 11, the performances of the ad hoc network for the last observed terrain are shown. The terrain is shaped in the form of a pyramid with a centrally positioned peak and sides that collide with the diagonals of the XY square projection (see Fig. 6). In this way, we have a division of the terrain into four parts. However, please note that these four parts do not overlap with the four clusters in the network. To the contrary, in every part of the pyramid we find halves of two clusters. But, as it can be seen from the figure, even using this arrangement instead of a perfect overlap of divisions we find great improvement of the performances of the ad hoc network no matter which type of clustering we observe. It is interesting that the maximum end-toend throughput is obtained for even higher offered load (4-5 Mbps). When performing a detailed analysis it can actually be shown that for lower loads the pyramid shows lower performances when compared to the flat terrain which is, of course, due to the difficulties in the communication establishment because of the blocking central top of the terrain. Also the clustering effect in the performance rise is a little bit lower here.

As summary results, the performances of the logically and physically clustered network for the observed terrains relatively to the flat terrain is shown on Fig. 12 for a more clear perspective of the network performances. The relative performances are calculated according to the following relation:

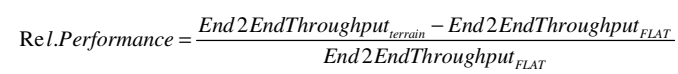

From all of these observations we can conclude that the terrain shape has great impact on the ad hoc network performances in the clustered and not clustered cases. When the clustering of the network coincides with the separation of the nodes in terms of LOS induced by the terrain shape we can expect performance improvements on a larger scale since the radio communication in the vicinity does not interfere with the one inside the cluster. Also, for terrains with a rising complexity, the obtained results greatly differ in both values and shapes of the functions when compared to the ones obtained for a flat terrain. This clearly shows the importance of taking the terrain into consideration when discussing the ad hoc network performances

\section{CONCLUSION}

In this paper the extension of the NS-2 simulator for using 3D terrain data in order to get more realistic results is introduced. The utilization of the Durkin's propagation model for clustered ad hoc network performance analysis in a more realistic environment is introduced.

The Durkin's method is very attractive because it can read in a digital elevation map and perform a site-specific propagation computation on the elevation data. It can produce results that are reported to be good within few $\mathrm{dB}$. Thus, these values are very good for our first order observation of some large scale factors that influence the ad hoc networks performances. The disadvantages are that it cannot adequately predict propagation effects due to foliage, buildings, other man-made structures and it does not account for multipath propagation other than ground reflection, so additional loss factors are often included. The propagation phenomenon that is modeled is LOS and diffraction from obstacles along the radial, and excludes reflections from other surrounding objects and local scatterers. The effect of this assumption is that the model is somewhat pessimistic in narrow valleys, although it identifies isolated weak reception areas rather well.

Of course, one of the reasons why the more realistic propagation models are omitted during the simulation stages is because they prolong the simulations several times over. Using the caching principle for our static simulations we were able to obtain results as fast as it would have been done for simulation scenarios that are defined over a flat regular terrain. However, we acknowledge the difficulties concerning the time needed to run the simulations for the mobile case. In order to obtain results for an identical environment that we used in our sets with the exception that the nodes are mobile and moving according to the random direction movement model with an average speed of $1 \mathrm{~m} / \mathrm{s}$ we needed 20 times more time on an average PC, and thus for mobile simulations we decided to use a grid computational environment that will allow parallel execution of several tcl scripts for NS-2.

When used for investigating the performances of clustered ad hoc networks, our results show that in certain terrain conditions the terrain can play a major role into lowering, but also and, boosting the ad hoc network performances. These results show that the presuming that the terrain will always impact on the performances towards its reduction isn't always true. Thus, the terrain configuration must be taken into consideration and, if the situation allows it, may be used to improve the network performances especially using the awareness of the natural clustering of the network on both, logical and physical, level.

As future work, we intend to expand our propagation model implementation with the more exact estimation of the cases when there are several obstacles on the line connecting the transmitter and the receiver. Using a software profiler, we intend to find the most time consuming tasks in the propagation model, and try to redo them in a way that is going to be more efficient so that we can do mobile simulations with more reasonable speed. Afterwards, we intend to offer our extensions for public use as an open source add-on to the NS-2 simulator which will be available online. 


\section{REFERENCES}

[1] Hekmat R. 2006. Ad-hoc Networks: Fundamental Properties and Network Topologies. Springer.

[2] Ozan, K., Tonguz, G., Ferrari. 2006. Ad Hoc Wireless Networks: A Communication-Theoretic Perspective, John Wiley \& Sons.

[3] Royer, E., Toh C., 1999. A Review of Current Routing Protocols for Ad Hoc Mobile Wireless Networks. IEEE Personal Communications 6 (2), April 1999, pp. 46-55

[4] Shan, W., Jian-xin, W., Xu-dong, Z., Ji-bo, W. 2006. Performance of anti-jamming ad hoc networks using directional beams with group mobility. IFIP International Conference on Wireless and Optical Communications Networks, 11-13 April 2006

[5] Baumann, R., Heimlicher, S., May, M. 2007. Towards Realistic Mobility Models for Vehicular Ad-hoc Networks. $26^{\text {th }}$ Annual IEEE Conference on Computer Communications IEEE INFOCOM 2007, 6-12 May 2007, Alaska, USA

[6] Edwards, R., Durkin, J. 1969. Computer Prediction of Service Area for VHF Mobile Radio Networks. Proceedings of the IEEE, Vol. 116, No. 9, pp. 1493-1500, 1969.

[7] NS-2 network simulator, Available: http://nsnam.isi.edu/nsnam/index.php

[8] The Guide to Geographic Information System, Available: http://www.gis.com

[9] Harmon, J. E., Anderson, S. J. 2003. The Design and Implementation of Geographic Information Systems. John Wiley \& Sons.

[10] U.S. Geological Survey National Mapping Division: Part 1 General, Standards for Digital Elevation Models

[11] U.S. Geological Survey National Mapping Division: Part 2 Specifications, Standards for Digital Elevation Models

[12] Terraform, Available: http://firedrake.org/terraform/
[13] Terragen, Available: http://www.planetside.co.uk/terragen/

[14] Rappaport, T. S., 2002. Wireless Communications: Principles and Practice, Prentice Hall, New York.

[15] Lee, W. C. Y. , 1985. Mobile Communications Engineering, McGraw Hill Publications, New York.

[16] Dadson, C. E. , Durkin, J. , Martin, E., 1975. Computer Prediction of Field Strength in the Planning of Radio Systems. IEEE Transactions on Vehicular Technology, Vol. VT-24, No. 1, pp. 1-7, 1975.

[17] Epstein, D., Peterson, D. W., 1953. An Experimental Study of Wave Propagation at $840 \mathrm{M} / \mathrm{C}$. Proceedings of the IRE, Vol. 41, No. 5, pp. 595-611, 1953.

[18] Perkins, C., 2003. Ad hoc On-Demand Distance Vector (AODV) Routing. Internet-Draft Experimental RFC 3561, july 2003.

[19] Watts, D. J., 2003. Small Worlds: The Dynamics of Networks between Order and Randomness, Princeton University Press

[20] Watts, D. J., 2003. Six Degrees: The Science of a Connected Age, W.W. Norton \& Company, New York

[21] Gladwell M., 2000. The Tipping Point, New York, Little, Brown

[22] Filiposka, S., Trajanov, D., Grnarov, A., 2007. Survey Of Social Networking And Applications In Ad Hoc Networks, ETAI, 2007

[23] Brown, J. S., Duguid, P., 2000. Social life of information, Harvard Business School Press

[24] Filiposka, S., Trajanov, D., Grnarov, A., 2006. Analysis of small world phenomena and group mobility in ad hoc networks. International Joint Conferences on Computer, Information, and Systems Sciences, and Engineering, Bridgeport, CT, USA, 2006 